\title{
Design and Development of a Two Channel Telemedicine System for Rural Healthcare
}

\author{
Mitrra Potheri Ramesh, Shruthi Balasubramanian, Varsha Vijayan*, \\ Geethanjali Balasubramanian, Mahesh Veezhinathan \\ Department of Bio-Medical Engineering, SSN College of Engineering, Kalavakkam, Chennai, India \\ Email: *varsha.bme@gmail.com
}

Received 2013

\begin{abstract}
In this work, an attempt has been made to design an Electro Cardio Gram (ECG) and Photo Plethysmo Gram (PPG) based telemedicine system for rural health care. In India as per the survey conducted by Indian Medical Society in the year 2009, it has been revealed that only $2 \%$ of the qualified doctors practice in rural areas. Also, according to the statistics taken by the World Health Organization, every year an estimated 17 million people die of various cardio vascular diseases. This paper introduces the transmission of ECG and PULSE from remote areas to the specialists' office. The ECG and PPG signals are acquired, digitized and processed using LabVIEW for detection of heart and pulse rate. These data are transmitted using two methods namely Web publishing tool and Shared Variables. The results confirm the transfer of data with less than millisecond delay.
\end{abstract}

Keywords: Telemedicine; ECG; LabVIEW; Web Publishing Tool; Shared Variables

\section{Introduction}

Telemedicine is the process of communication of healthcare related information from one place to another in the form of interactive audio visual media. The rapid advancements in information and communication technology can be used to bridge the rural urban divide. This technique proves to be very important during life threatening situations and in places with lack of availability of specialists.

Various methods of transmission of physiological data have been carried out previously. One such system uses Time Division Multiplexing (TDM) to transmit data via Bluetooth [1]. Another system uses a wearable vital sign monitor that consists of a transmitter. It transmits the information via a Home Gateway using a proprietary protocol nRF24xx, to the central station [2]. The transmission has also been achieved using RF transmitter and receiver with slotted ALOHA for access by multiple users [3].

This paper deals with the acquisition, processing and transmission of ECG and PPG signals from the remote computer to the client using Web publishing tool and Shared Variables. Hence, the diagnosis of a rural patient can be facilitated by a city doctor with access to a computer installed with LabVIEW and Internet. Further, it eliminates the need for the conventional transmitter-receiver with limited range. The other applications include

${ }^{*}$ Corresponding author. transmission of vital details from ICU to the doctor's cabin and future developments can be done in transmitting the parameters to specialist who is based anywhere in the world.

\section{Materials and Methods}

\subsection{Experimental Setup}

The ECG-based telemedicine system [4] mainly consists of the following five parts-1) Data acquisition system; 2) Amplification of the acquired signals using hardware modules; 3) Physiological signal reception in computer; 4) Signal processing and display; 5) Web publishing tool and Shared Variables for viewing the signals on a browser at a remote computer. The signals can be viewed and controlled from both the remote computer (server) and the clients' side.

\subsection{Data Acquisition System}

The ECG data is acquired from surface electrode using Standard Recording Protocol. The acquired ECG is then amplified using a hardware module with IC AD620. The pulse signal is acquired using a Pulse Oximeter and amplified using IC LM324. The amplified signals are then given as input to the three part NI-ELVIS system, the NI-ELVIS workstation (prototype board) that interfaces with the NI-DAQ (data acquisition) device and LabVIEW software. NI ELVIS has a sampling rate of 1.25 
MegaSamples/second for single channel and an aggregate of 1.00 MegaSamples/second [5] for multichannel. The outputs of ECG amplifier and Pulse amplifier channel are AI-0, AI-1 of NI-ELVIS respectively. NI-DAQ captures the ECG and pulse signals for analysis in LabVIEW.

\subsection{Signal Analysis}

Instrumentation amplifier AD620 is used for amplifying the acquired ECG. The choice of AD620 for this analysis is due to its low noise, low input bias current and low power and the use of a single external resistor for Gain variation from 1 to 10,000 [6]. The gain-setting resistor $\left(\mathrm{R}_{\mathrm{G}}\right)$ determines the gain, given by Equation (1).

$$
\mathrm{G}=1+\left\{(49.4) \mathrm{k} \Omega / \mathrm{R}_{\mathrm{G}}\right\}
$$

The CMRR for gain $=10$ is about $100 \mathrm{~dB}$. IC LM324 is used for pulse amplification. The analog signals are then processed in LabVIEW.

\subsubsection{ECG Analysis}

ECG acquired from healthy subjects using surface electrodes is preprocessed prior to detailed analysis. Initially, raw ECG is preprocessed for noise and baseline drift removal. This is done using Wavelet Denoising tool. Daubechies 6 (db6) [7] wavelet is suitable because its shape resembles that of ECG. For better balance between smoothness and accuracy when compared to Discrete Wavelet Transform (DWT), Undecimated Wavelet Transform (UWT) [8] of level 8 is applied. The choice of this level is because the noise removal efficiency is proportional to the increase in levels. In addition, a sampling rate of $1000 \mathrm{~Hz}$ is used; level 8 accounts for $7 \mathrm{~Hz}$ which falls within QRS complex range. Pan Tompkins [9] algorithm is then implemented for Heart Rate estimation. Finite Impulse Response band pass filter of order 30, with cut off frequency of $5-11 \mathrm{~Hz}$ is used for allowing QRS complex. The filtered ECG is then differentiated and squared to enhance the high frequency R-waves present. The peaks are then smoothened using movingwindow integrator which is essential to avoid multiplecounting. Tone measurements block is used to compute the frequency and eventually, the time interval and heart rate are calculated and displayed. Two abnormal conditions prominent in ECG viz. Bradycardia (below 30 beats) and Tachycardia (above 100 beats) are simulated using the rate adjustable ECG simulator Cardiosim. Boolean indicators for these conditions are applied. The flow chart of the process is shown in the Figure 1.

\subsubsection{Pulse Measurement}

Pulse signals are acquired using infrared LED pulse oximetry type sensor. LM 324 amplifier is used for amplifying the pulse signal acquired from subjects. The amplified signal is filtered using Moving Average Rectangular Smoothing filter. Tone measurements tool is used for measuring the amplitude and frequency. Time period and pulse rate are determined from the frequency obtained. Flow chart for pulse analysis is shown in Figure 2.

The block diagram of the entire system is as shown in Figure 3.

The block diagram of the entire system is as shown in Figure 4.

\section{Telemedicine Module}

The acquired data has been transmitted using two methods, Web Publishing tool and Shared Variables available in

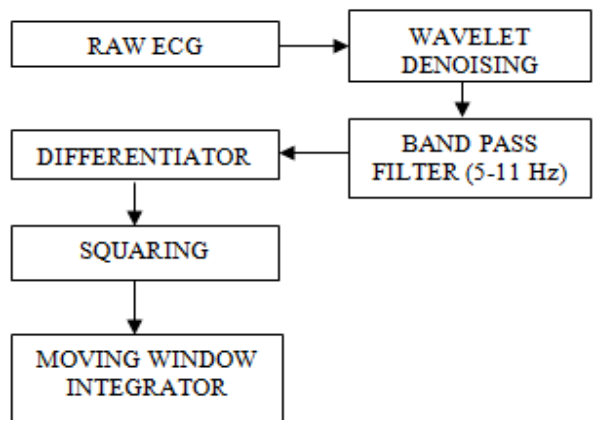

Figure 1. Block diagram for heart rate detection.

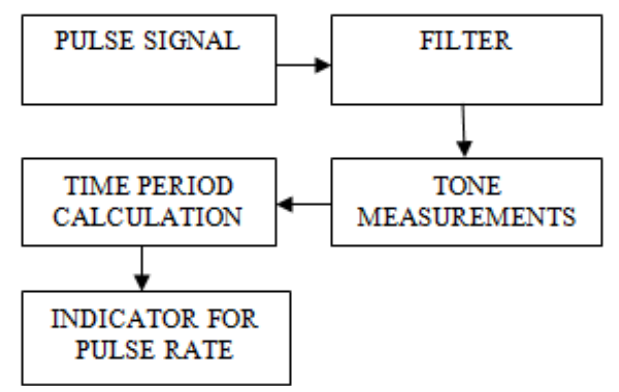

Figure 2. Block diagram for pulse rate detection.

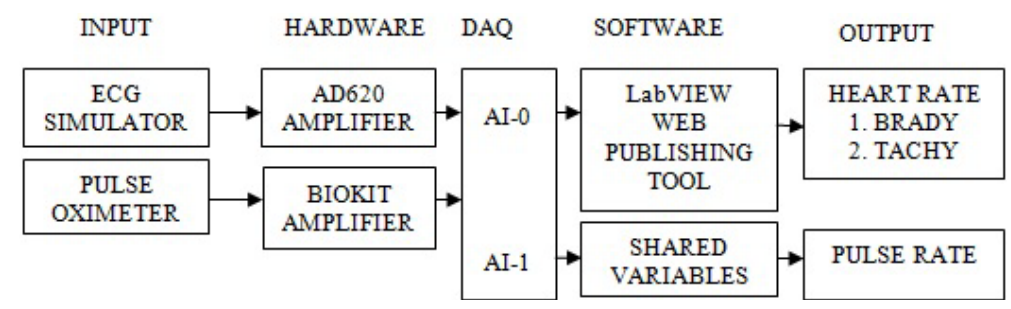

Figure 3. Block diagram of telemedicine system. 
LabVIEW. The above stated methods utilize TCP/IP to transfer the data via the Internet.

\subsection{Web Publishing Tool}

LabVIEW has a built in Web Server that can be used to publish front panel images by creating a HTML document. This tool produces Joint Photographic Experts Group (JPEG) and Portable Network Graphics (PNG) image formats of the front panel. There are three modes for displaying the images of the front panel, "snapshot" mode for publishing static images, "monitoring" mode for publishing animated images with a configurable refresh interval and the "embedded" mode which enables the viewing and control of the webpage by the end user [10].

The Front Panel of the real time ECG and Pulse monitoring system is selected in the embedded mode in the web publishing tool to allow viewing and control by the end user. Additional information other than the inclusion of front panel can be specified before the generation of the HTML document. The URL of the generated HTML document is then created. This URL can be used in other computers connected via the LabVIEW Web Server to view the front panel. Also, the client can request for access to control the Front panel.

\subsection{Shared Variables}

Shared Variables available in LabVIEW is used to share data across the web using NI Distribution System Manager. This method necessitates the availability of the Shared Variable engine in both the remote and client computer. From among the three types of Shared Variables that can be used viz. Single process, Network and
Time Triggered, Network type is used in this work. Network Shared Variables are used to read and write data via Ethernet, using NI Publish and Subscribe Protocol (NI-PSP).

Shared variable engine is used to host the variables on network after deployment. The shared variables can be transferred in a local network connected by a LAN and Wi-Fi. Two separate variables are to be created, one on the server side and the other on the client side. The variables can be viewed using NI Distribution System Manager and can be continuously refreshed. Numeric data along with its trend can be shared using the above process.

\section{Results and Discussion}

The ECG and PPG VIs have been combined into a single Telemedicine System using Sub-VIs in LabVIEW. Figure 5 shows the front panel of the consolidated system.

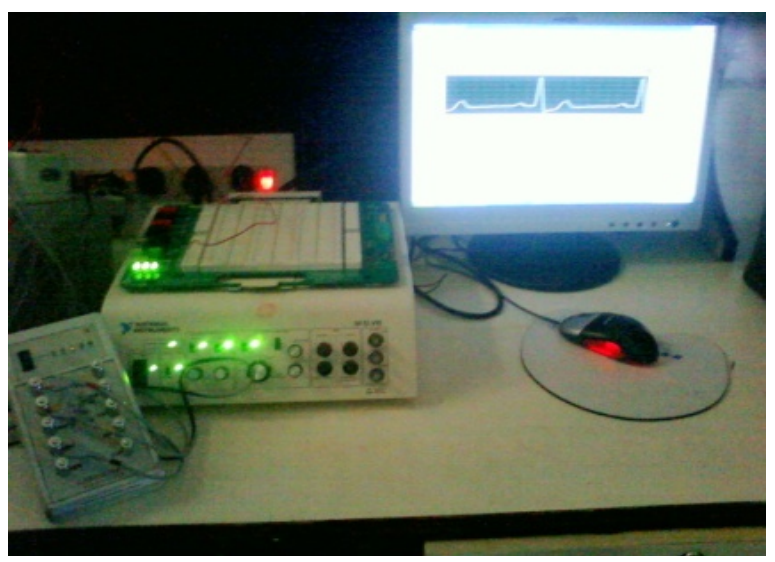

Figure 4. Simulation for abnormal condition.

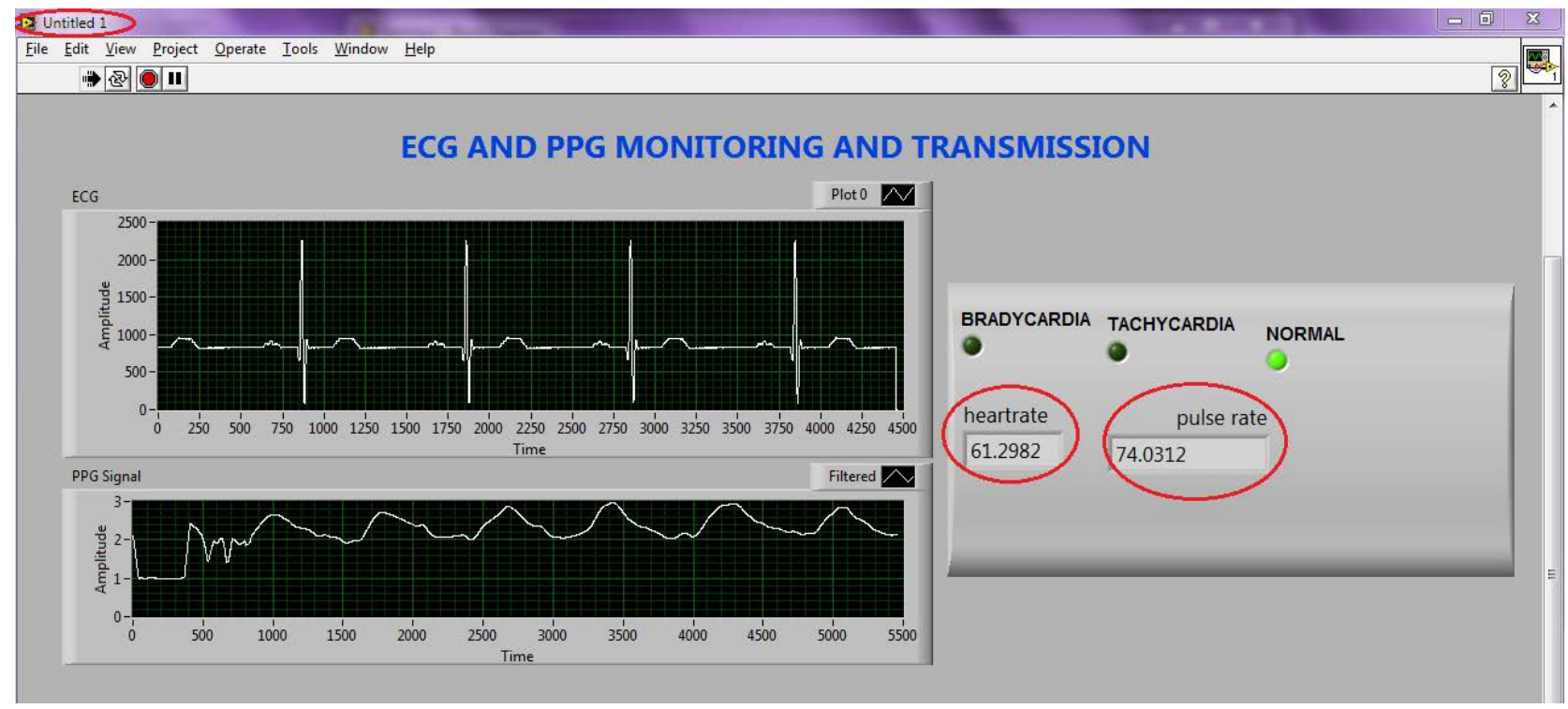

Figure 5. Final front panel of telemedicine system (TRAN- SMISSION SIDE). 
Figure 6 shows the output in a web browser obtained using Web Publishing Tool. The client computer displaying the output of numerical value of Heart Rate transmission using Shared Variables is shown in Figure 7. Similarly, the Pulse Rate transmission output using Shared Variables can be seen by altering the view in the same window. The output of Web Publishing Tool is viewed using any web browser with the URL of the VI. The numerical values such as the Heart and the Pulse Rates are transmitted efficiently using Shared Variables, thus saving bandwidth.

The results obtained are compared with previously existing systems. One such system [11] uses TCP/IP programming in LabVIEW to transmit a single channel of ECG. This system proves to be more efficient in terms of transmitting multiple parameters with minimum delay. The proposed method deploying Shared Variables saves more bandwidth when compared to the previous system [4]. The range of Wi-Fi used is $100 \mathrm{~m}$ radius [12], which is better when compared to Bluetooth [1] range of $10 \mathrm{~m}$.

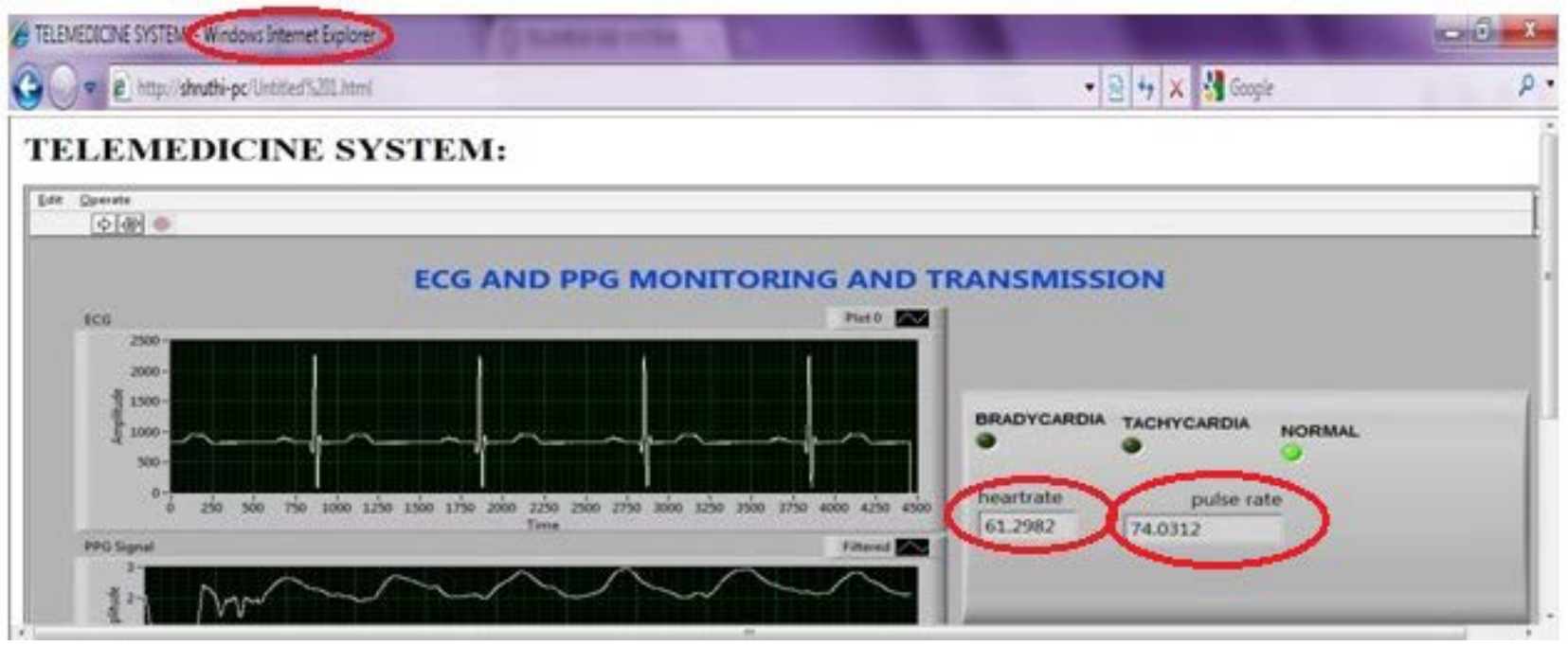

Figure 6. Web browser showing the front panel (RECEIVING SIDE).

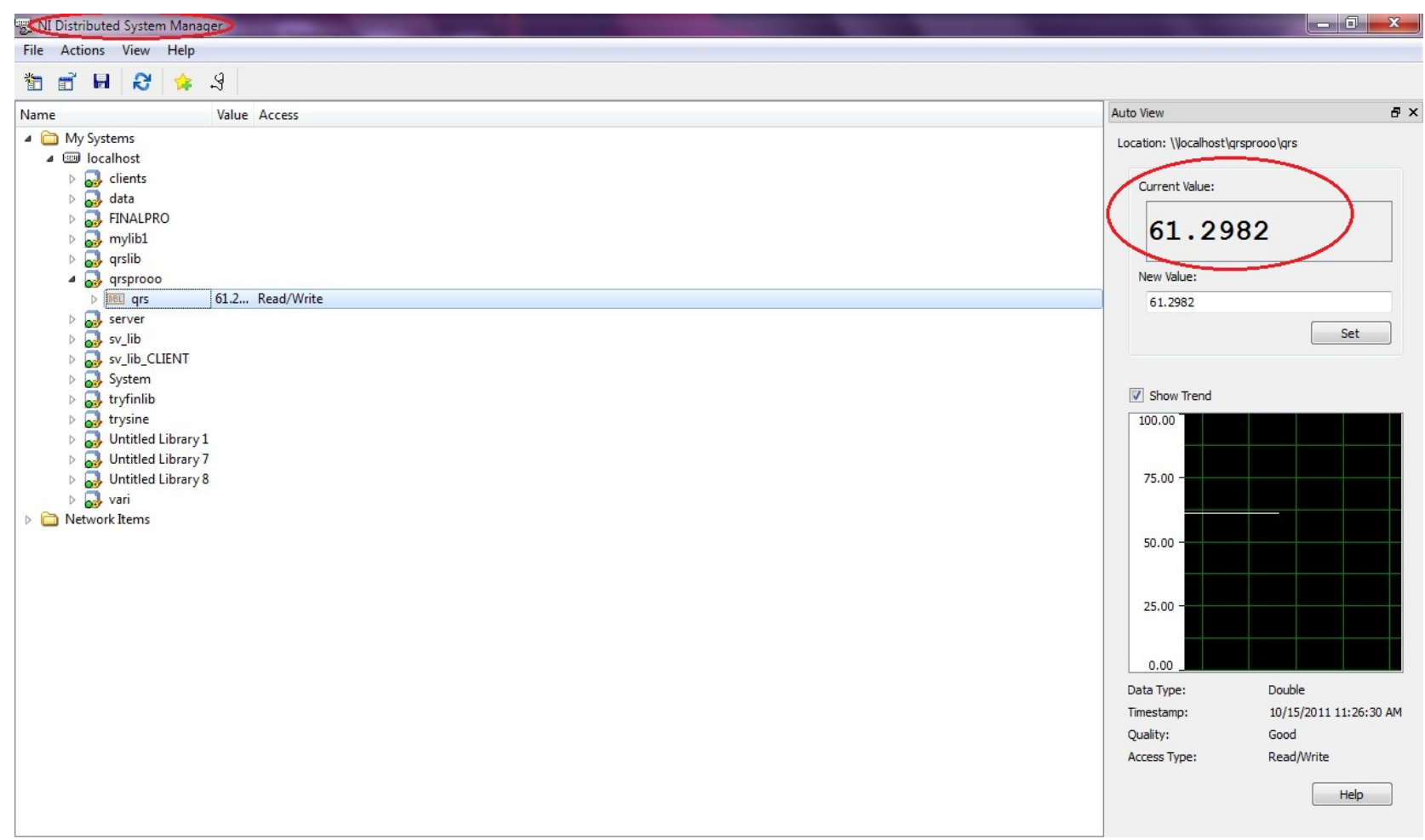

Figure 7. Transmitting heart rate using shared variables. 
Also, Web Publishing tool output can be published on any system with LabVIEW Web Server. Since the proposed system uses LabVIEW for signal processing, it is easy to use and maintain with low energy consumption, unlike the system [2] which uses a high end ARM processor.

\section{Conclusion}

The ECG and Pulse signals were successfully acquired, preprocessed and transmitted using the transmission methods available in LabVIEW viz. Web Publishing tool and Shared Variables. The output of Web Publishing Tool is viewed using any web browser and numerical values such as the Heart and the Pulse Rates are transmitted efficiently, thus saving bandwidth. The potential usefulness of this two channel low-cost, low-power telemedicine system is aimed for improving rural health monitoring. Further enhancement of this work would be to extend it to a multiple physiological parameter transmission module with provision for storage of waveforms and other data.

\section{REFERENCES}

[1] M. J. Deghani, A. R. Shahabina and A. A. Safari, "Implementation of wireless based Bluetooth technology for Biosignal Monitoring," World Applied Sciences Journal, Vol. 3, 2010,pp. 287-293.

[2] X. B. Shen, J. P. Zeng and T. D. Hin, "Remote Healthcare Monitoring System," International Conference on Biomedical Engineering and Informatics, Vol. 3, 2010, pp. 1901-1905.

[3] H.-L. Chan, C.-Y. Lu, C.-H. Liu and M.-A. Lin, "LowPower Wireless Transmission of Biosignals Using the Stotted ALOHA Mechanism,” IEEE International Conference on Systems, Man and Cybernetics, Vol. 1, 2006, pp. 513-517.
[4] X. Tan, X.-M. Guo, M. Cheng, Y. Yan, College of Bioengineering, Chongqing University, Chongqing, "Wireless Telemedicine Physiological Monitoring Center Based on Virtual Instruments," The 1st International on Bioinformatics and Biomedical Engineering, Wuhan, 6-8 July 2007, pp. 1157-1160.

[5] National Instruments, "Integrated Suite of 12 Instruments for Interative, Cost-Effective, Multidisciplinary, HandsOn Learning [Data File],” 2008.

http://www.addlink.es/pdf/AGDWeb1711.pdf

[6] Low Cost Low Power Instrumentation Amplifier AD620 [Data File]. 1999.

http://www.stanford.edu

http://www.stanford.edu/class/ee122/Parts_Info/datasheet s/ad620.pdf

[7] P. Sauravh and M. Madhuchhanda, "Detection of ECG Characteristic Points Using Multiresolution Wavelet Anaylsis Based Selective Coefficient Method,” Elsevier Measurement, Vol. 43, No. 2, 2010, pp. 255-261.

[8] N. Akshay, N. A. V. Jonnabhotla, N. Sadam and N. D. Yeddanapudi, "ECG Noise Removal and QRS Complex Detection Using UWT," IEEE International Conference on Electronics and Information Engineering (ICEIE), Kyoto, 1-3 August 2010, pp. 438-442.

[9] M. R. Rangaraj, "Biomedical Signal Analysis-A Case Study Approach,” IEEE Press, Calgary, 2002, pp. 187190.

[10] P. F. Li and L. H. Nie, "Remote control Laboratory based on LabVIEW,” 2nd International Conference on Intelligent Computation Technology and Automation, Vol. 4, 2009, pp. 84-87.

[11] H. Shekhar, J. S. J. Kumar, V. Ashok and A. V. Juliet, “Applied Medical Informatics Using LabVIEW," 2nd International Conference on Intelligent Systems, Modelling and Simulation, Vol. 2, 2010, pp. 198-203.

[12] B. Sidhu, H. Singh and A. Chhabra, "Emerging Wireless Standards-WiFi, ZigBee and WiMAX,” World Academy of Science, Engineering and Technology, Vol. 25, 2007, pp. 1345-1349. 